\title{
Tension-Optimized Highly Nonlinear Fiber for Parametric Applications
}

\author{
Carl Lundström ${ }^{(1)}$, Evgeny Myslivets ${ }^{(2)}$, Andreas O.J. Wiberg ${ }^{(2)}$, Nikola Alic ${ }^{(2)}$, \\ Stojan Radic ${ }^{(2)}$, Magnus Karsson ${ }^{(1)}$, Peter A. Andrekson ${ }^{(1)}$ \\ (1) Photonics Laboratory, Department of Microtechnology and Nanoscience, \\ Chalmers University of Technology, S-412 96, Gothenburg, Sweden, carl.lundstrom@chalmers.se \\ (2) Department of Electrical and Computer Engineering, University of California at San Diego, La Jolla, \\ CA 92093, USA
}

Abstract We investigate the Brillouin and dispersive properties including localized measurements of nonlinear fibers spooled with tension gradients and discuss their application in parametric devices.

\section{Introduction}

Since their introduction, Highly Nonlinear Fibers (HNLFs) have proved to be a successful platform for many applications based on fourwave mixing (FWM) and other nonlinearities. Examples include parametric amplifiers ${ }^{1}$, wavelength (WL) converters, and many more. HNLFs have very low attenuation compared to other nonlinear optical media, giving a very competitive nonlinearity coefficient over attenuation figure-of-merit ${ }^{2}$. However, HNLFs are hampered by the Stimulated Brillouin Scattering (SBS) effect, which limits the amount of power that can be injected into the fiber, and thereby the achievable nonlinear phase shift. Often, this is overcome by broadening the highpower wave (the pump) spectrally using e.g. a phase modulator (PM) to more than the SBS bandwidth (which is typically tens of $\mathrm{MHz}$ ). This method can increase the threshold many times; however for many applications it is undesirable to have a spectrally broad pump. FWM-based WL converters suffer, since the generated idler will carry twice the pump phase, and in phasesensitive amplification based on FWM, spectrally broad waves can be very problematic since they require very careful phase control of the interacting waves.

There are a number of passive methods that can be used to suppress the SBS in nonlinear fiber devices by dividing the medium into independent (in terms of SBS) sections either by using segments with different SBS downshift frequency or by periodically blocking the backscattered wave with isolators. A problem with this method is the additional losses introduced due to the splicing of different fibers, and many isolators are required to substantially increase threshold. Another method is to use a single fiber and introduce a non-uniformity along the fiber, either during manufacturing or afterwards. Unfortunately, when non-uniformity is introduced that shifts the SBS frequency, zero-dispersion wavelength fluctuations (ZDWF) is typically also introduced. In FWM applications this is undesirable since it limits the phasematching and thus the bandwidth of such devices. Both temperature ${ }^{3}$ and tension gradients $^{4}$ have been demonstrated to successfully broaden the SBS spectrum of nonlinear fibers, but investigation of the effect on the dispersion has been limited. Unlike specially manufactured fibers and segmentation of the HNLF, these methods are also reconfigurable, providing a practical method for lab use. We have previously shown that a fiber tension gradient can be used to reduce the dispersion fluctuations of an $\mathrm{HNLF}^{5}$, and in Ref. [6], a specially designed dispersion-decreasing HNLF was presented, giving low dispersion variations after applying a tension gradient. Here, we show that fiber tension can be a practical way to suppress SBS also for conventional HNLFs, using a commercially available spooling machine and we for the first time study the trade-off between SBS spectrum broadening and ZDW shift using localized measurements. We show that the SBS-limited parametric efficiency can be significantly increased while maintaining reasonable parametric bandwidths.

\section{Theory}

Let us consider two main classes of FWM devices: low-gain, (e.g. WL conversion or multicasting), where the target is often transparency and the efficiency is quadratically dependent on available pump power, and highgain, (e.g. parametric amplification) where the efficiency is exponentially dependent on available pump power. Increasing the threshold will have a much larger effect in the latter case; unfortunately the SBS-threshold of conventional HNLFs is usually quite far from this regime.

By using the matrix method ${ }^{7}$, the effect of ZDWF can be studied. A typical value of the SBS-limited FWM conversion efficiency in HNLFs is about $-15 \mathrm{~dB}$. Fig. 1 shows the SBSlimited conversion efficiency spectrum in an HNLF with no ZDWF (pump WL: $\lambda_{p}=Z D W+0.5$ 
$\mathrm{nm}$ ), and that of an HNLF with a $7.4 \mathrm{~dB}$ increased threshold and $11.5 \mathrm{~nm}$ of linear ZDWF along the fiber. Obviously, $\lambda_{p}$ needs to be changed as the ZDW is shifted. In the latter case, efficiency slightly above transparency can be reached, but the maximum $3 \mathrm{~dB}$-bandwidth is reduced from more than $130 \mathrm{~nm}$ to about $75 \mathrm{~nm}$ at a $\lambda_{p}$ of $1553.5 \mathrm{~nm}$. Also shown is a case $\left(\lambda_{p}=1558 \mathrm{~nm}\right)$ when the pump is in the anomalous dispersion regime throughout the fiber - slightly higher efficiency is then attained.

In the high-gain case, we can compare the gain spectrum of the same HNLF with the threshold increased 15 times, either with 11.5 $\mathrm{nm}$ of linear ZDWF or without any ZDWF (e.g. by using pump PM). This is shown in Fig. 2, and approximately the same maximal gain (about 17 $\mathrm{dB}$ ) can be obtained in both cases, albeit at a smaller bandwidth in the ZDWF case. The pump needs to remain in the anomalous dispersion regime. From these results, it is clear that 11.5 $\mathrm{nm}$ of linear ZDWF by no means excludes an HNLF to be useful in neither low-gain nor highgain parametric applications.

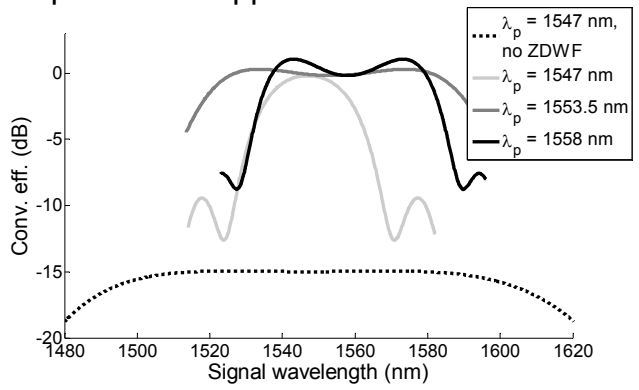

Fig. 1: Calculated conversion efficiency spectra in a typical HNLF with no ZDW fluctuations pumped at threshold (ZDW=1546.5 nm, dotted line), and for an HNLF with a tension gradient giving $7.4 \mathrm{~dB}$ increased threshold and $11.5 \mathrm{~nm}$ of ZDWF. (1546.5-1558 nm, solid lines).

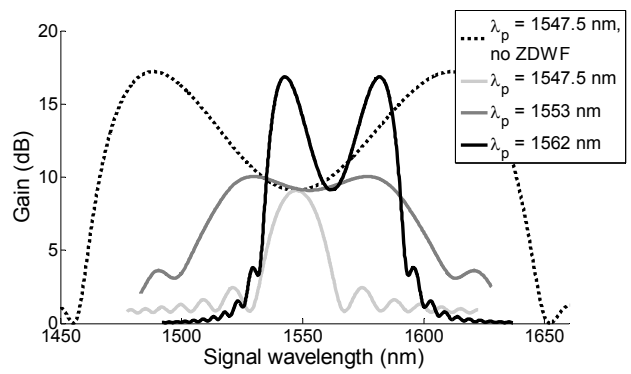

Fig. 2: Calculated parametric gain spectra in an HNLF with no ZDW fluctuations (ZDW=1546.5 nm, dotted line), and for $11.5 \mathrm{~nm}$ of ZDWF (1546.5-1558 $\mathrm{nm}$, solid lines). The pump power was the same in all cases.

\section{Experiment}

A conventional $100 \mathrm{~m}$ HNLF with a nonlinearity coefficient of about $10 \quad(\mathrm{~W} \cdot \mathrm{km})^{-1}$ was investigated. To spool it with different and/or varying tension, a commercial spooler for optical fibers was used. It had an hysteresis brake to control fiber tension. To measure the SBS spectrum of the HNLF a simple selfheterodyning method was used, where light from a laser was split, amplified and injected into the fiber. The resulting backscattered power is mixed with the remaining light on a photodiode and the spectrum observed on an electrical spectrum analyzer. The average dispersion of the HNLF was then measured with a commerical dispersion analyzer (Advantest Optscope).

Fig. 3 shows the measured SBS spectrum of the HNLF when it was spooled using different brake torques, from no torque to the maximal available (but kept the same along the entire fiber). Clearly, different levels of tension shifted the entire SBS spectrum upwards in frequency, but left the spectral shape unaffected, as expected. We were able to shift the center frequency up to about $300 \mathrm{MHz}$, which can be compared with the SBS spectrum $10 \mathrm{~dB}$ bandwidth of about $20 \mathrm{MHz}$. However, as shown in Fig. 4, the average zero-dispersion wavelength of the fiber also shifted from about $1546.5 \mathrm{~nm}$ (no torque) to $1558 \mathrm{~nm}$ (max. torque. The dispersion slope remained unchanged. From this result, we can expect that it is possible to spool the fiber with step-wise varying tension to achieve a broad SBS spectrum and zerodispersion wavelength variations of no more than about $11.5 \mathrm{~nm}$. The resulting SBS spectra of three attempts are shown in Fig. 5. Some trial-and-error was required to get a relatively flat spectrum, which gives the maximal SBS threshold increase. This also shows the reconfigurability of the spooling.

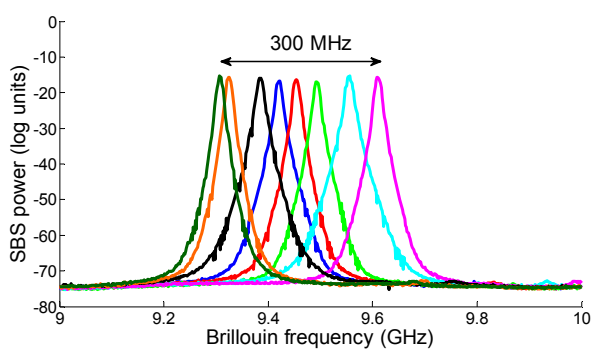

Fig. 3: Measured SBS spectra of the HNLF when spooled with different tension levels.

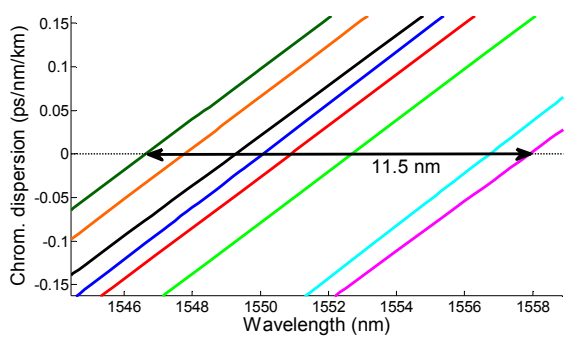

Fig. 4: Measured dispersion curves of the HNLF when spooled with different tensions levels. 


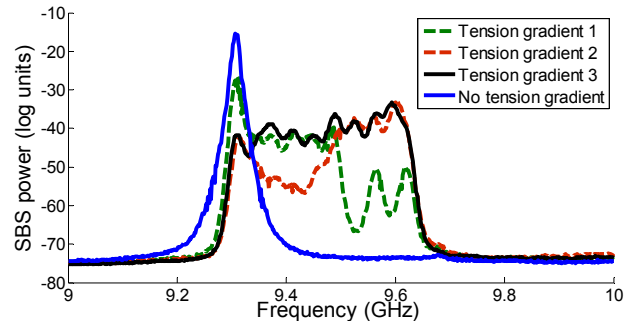

Fig. 5: Measured SBS spectra of the HNLF when spooled with different tension gradients.

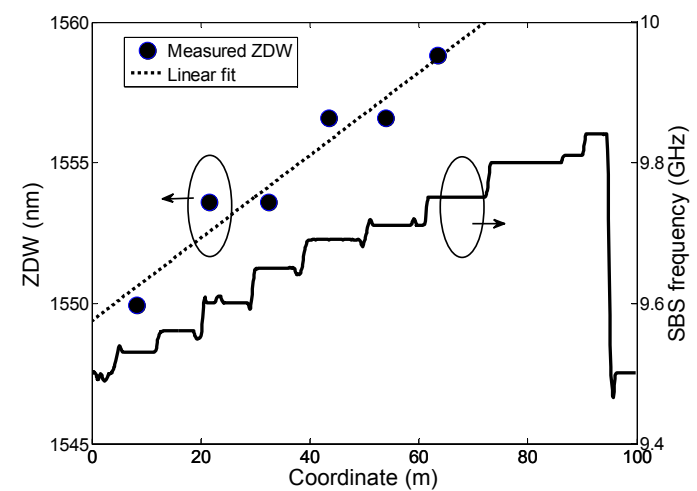

Fig. 6: Measured local ZDW and measured local SBS downshift in the HNLF with tension gradient 3.

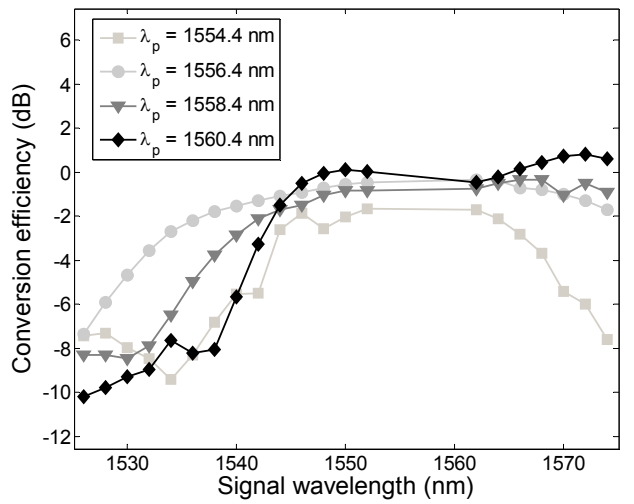

Fig. 7: Measured FWM conversion efficiency in the HNLF with tension gradient 3 at four different pump wavelengths. Without tension gradient, the conversion efficiency was below $-10 \mathrm{~dB}$.

Tension gradient 3 used 11 different brake settings and gave the best result with a relatively uniform spectrum. The SBS $10 \mathrm{~dB}$-bandwidth is increased almost 15 times to about $300 \mathrm{MHz}$. For a perfectly uniform tension gradient, a threshold increase of about 15 times would be expected for such a broadening, but in the present case the threshold increase was less. With tension gradient 3 applied, we subsequently retrieved the local SBS frequency (1m step-length) and local ZDW (about $11 \mathrm{~m}$ step-length, only about two thirds of the fiber measured) using the counter-colliding scanning method $^{8}$, which allows for a reconstruction of the ZDW map as well as a mapping of the Brillouin frequency shift in the fiber. Fig. 6 shows both the SBS frequency shift and ZDW maps. In the SBS map, the 11 different tension levels can clearly be identified. While the ZDW mapping could not resolve all 11 levels, it showed ZDW variations of about $9 \mathrm{~nm}$ in the first two thirds of the fiber, which corresponds relatively well with our prediction of about $11.5 \mathrm{~nm}$ ZDW variation along the fiber. The ZDW variation without a tension gradient was about $\pm 1 \mathrm{~nm}$.

The SBS threshold with strain gradient 3 was about $29.5 \mathrm{dBm}$. At this pump power, the FWM conversion efficiency was measured for four different pump wavelengths, with the results shown in Fig. 7. While the broadest $3 \mathrm{~dB}$ bandwidth $(49 \mathrm{~nm})$ was achieved for a pump wavelength of $1556.4 \mathrm{~nm}$, a longer pump wavelength of $1560.4 \mathrm{~nm}$ achieved a higher maximum efficiency, slightly above transparency, as the pump remained in the anomalous dispersion regime throughout the HNLF. The measured results correspond well with the calculated in Fig. 1.

\section{Conclusions}

We have measured the effect of tension on HNLFs in terms of SBS and dispersion, including localized measurements. We showed that despite a large increase in SBS spectral width and thus threshold, the increase in ZDWF can be small enough for the HNLF to be usable in both low- and high-gain parametric applications. Furthermore, the fiber can be respooled with different tension profiles and the method requires only a standard commercial spooling machine. It is expected that a more uniform tension gradient could increase the threshold further, enabling high parametric efficiency. The behavior of different types of HNLFs under tension and the effect on fiber birefringence remain a topic for further studies.

\section{Acknowledgements}

Sumitomo Electric Industries, Ltd. is acknowledged for providing the HNLF. This work was supported in part by the Swedish Foundation for International Cooperation in Research and Higher Education (STINT) and also by the Swedish Research Council (VR).

\section{References}

[1] J. Hansryd et al., JSTQE 8, 506 (2002)

[2] M. Hirano et al., JSTQE 15, 103 (2009)

[3] J. Hansryd et al., JLT. 19, 1691 (2001)

[4] J.M.C. Boggio et al., JLT. 23, 3808 (2005)

[5] E. Myslivets et al., PTL. 211807 (2009)

[6] M. Takahasi et al., JLT. 28, 59 (2010)

[7] M. Farahmand et al., OE. 12, 136 (2004)

[8] E. Myslivets et al., JLT. 27, 364 (2009) 